\title{
Restoring Movement in Paralysis with a Bioelectronic Neural Bypass Approach: Current State and Future Directions
}

\author{
Chad E. Bouton \\ Feinstein Institute for Medical Research, Northwell Health, Manhasset, New York 11030 \\ Correspondence: cbouton@northwell.edu
}

Bioelectronic medicine is a rapidly growing field that explores targeted neuromodulation in new treatment options addressing both disease and injury. New bioelectronic methods are being developed to monitor and modulate neural activity directly. The therapeutic benefit of these approaches has been validated in recent clinical studies in various conditions, including paralysis. By using decoding and modulation strategies together, it is possible to restore lost function to those living with paralysis and other debilitating conditions by interpreting and rerouting signals around the affected portion of the nervous system. This, in effect, creates a bioelectronic "neural bypass" to serve the function of the damaged/degenerated network. By learning the language of neurons and using neural interface technology to tap into critical networks, new approaches to repairing or restoring function in areas impacted by disease or injury may become a reality.

$T^{\text {timen }}$ raumatic injuries, especially those involving the brain and spinal cord, can be particularly difficult to treat. Researchers have attempted to develop pharmaceutical and stem cell approaches to promote healing in spinal cord injuries, but have been met with limited success. For injuries that have led to motor impairment, assistive and rehabilitative technologies using neuromuscular stimulation and robotics have been developed. Robot-assisted therapy has been shown to be effective in stroke patient recovery, particularly for upper-limb rehabilitation (Volpe et al. 2008; Lo et al. 2010). In the case of traumatic brain injury, closely monitoring the patient multiple parameters in the brain is crucial. To allow this, multimodal sensing technologies have been re- cently developed for sensing key parameters in the brain simultaneously, providing valuable information to the clinical team during recovery (Li et al. 2009). Also, stimulation strategies such as trigeminal nerve stimulation have been studied with the goal of improving traumatic brain injury outcomes (Chiluwal et al. 2017).

In cases in which disease or injury has led to complete paralysis, such as in spinal cord injury (SCI), stroke, and motor neuron disease, braincomputer interface (BCI) technology has been of particular interest. BCI technology allows signals in the brain to be recorded and decoded to determine the user's thoughts, which can allow a paralyzed or locked-in patient to communicate, control devices, and, when combined with neu-

Editors: Valentin A. Pavlov and Kevin J. Tracey

Additional Perspectives on Bioelectronic Medicine available at www.perspectivesinmedicine.org

Copyright (C) 2019 Cold Spring Harbor Laboratory Press; all rights reserved; doi: 10.1101/cshperspect.a034306

Cite this article as Cold Spring Harb Perspect Med 2019;9:a034306 
romuscular stimulation technology, regain volitional movement. To create a BCI system, several technologies are required, including brain electrodes, machine learning algorithms to decode intentions, and a device to be controlled (e.g., a computer, robotic arm, neuromuscular stimulators). To decode brain activity, it is critical to understand how information is encoded in the brain so that electrical activity can be monitored and decoded to determine a patient's intent (e.g., to communicate, move, etc.). Early studies were conducted in the 1960 s to determine how sensorimotor information is encoded, which paved the way for BCIs (Evarts 1968; Perkel and Bullock 1968). Later, neural modulation patterns were studied during active and passive movements (Fetz et al. 1980) and force-related neurons were identified (Cheney and Fetz 1980). As electrode technology developed, populations of neurons could be studied, and it was discovered that neurons in the motor cortex have a "preferred" direction, known as "directional tuning” (Georgopoulos et al. 1986). As computer technology further developed, our understanding of modulation patterns developed (Warland et al. 1997; Donoghue et al. 1998). All of these important studies laid the groundwork for subsequent advancements in developing BCI technology for debilitating conditions.

One of the critical components of bioelectronic medicine that uses BCI devices is the neural interface. This interface provides a way to sense, record, and modulate neural signals in nerves and in the brain. Recent advances have been made in flexible nerve cuff electrodes allowing acute and chronic recordings (Caravaca et al. 2017). Multielectrode arrays for the brain have been under development for many years with researchers facing many challenges. Initially, electrodes were made by hand in the laboratory, which limited the number of recording sites each had. Microfabrication techniques were later developed and progress accelerated. Researchers at University at Utah began to use etching techniques to form electrode arrays from a solid piece of silicon. Precise electrode "spikes" could be formed with uniform spacing. Encapsulation methods for this style of electrode arrays were also refined for chronic applications in neural disorders (Hsu et al. 2009). Researchers at the University of Michigan also developed brain and nerve electrodes through a thin-film process to make flexible electrodes, and chronic brain recordings have been achieved (Vetter et al. 2004). Electrocorticography (ECoG) electrodes can also be used for recordings on the surface of the brain. These electrodes typically are not intended for capturing single-unit action potentials and the spatial resolution is lower than the Utah and Michigan style electrodes.

Once neural signals are accessed through a neural interface, they must be processed and deciphered to extract useful information. In the late 20th century, a branch of artificial intelligence, known as machine learning, began to accelerate (Shavlik and Dietterich 1990; Michie et al. 1994). Computers were becoming more powerful and the interest in studying signals recorded in the brain was growing. The rate at which neurons "fire," conveys useful information that can be used to determine the user's intentions such as moving a cursor on a computer screen in a particular direction. This new area of neural decoding was born and many researchers were trying to achieve what initially seemed impossible-read someone's mind.

Researchers studied brain activity in primates and linked their thoughts to robotic arms (Chapin et al. 1999; Wessberg et al. 2000; Serruya et al. 2002; Taylor et al. 2002; Carmena et al. 2003; Lebedev et al. 2005). It was shown that these activity patterns were related to movement in three-dimensional space (Taylor et al. 2002; Velliste et al. 2008). Taking this a step further, primates were able to regain volitional control over their own (temporarily paralyzed) limbs (Moritz et al. 2008; Ethier et al. 2012). Clinical studies also were launched to determine whether these technologies and methods could be used to restore speech (Kennedy and Bakay 1998) or be used to assist patients living with paralysis caused by SCI, stroke, and amyotrophic lateral sclerosis (ALS) interface with their computers, directly with their minds (Hochberg et al. 2006; Bouton 2009). Later, control of robotic arms was then shown in a clinical study (Hochberg et al. 2012; Collinger et al. 2013). 
Finally, functional movement in a paralyzed human was recently shown through the use of a neural interface implanted in the brain and neuromuscular stimulation (Bouton et al. 2016).

As the field of bioelectronic medicine continues to grow rapidly, multidisciplinary teams work closely to generate preclinical mechanistic insight, define new options in disease diagnosis and treatment, and validate their clinical relevance in the context of various disorders including paralysis. Modulating neural pathways, such as the vagus nerve-based inflammatory reflex, has a positive effect in chronic inflammatory conditions including rheumatoid arthritis and Crohn's disease (see Tracey 2002, 2015; Pavlov and Tracey 2015). Furthermore, listening in on neural pathways and decoding the acquired signals can give us insight into changes in inflammatory proteins and other important substances in our bodies, potentially leading to earlier and better diagnostics one day (see Bouton 2017; Olofsson and Tracey 2017; Zanos et al. 2018). We need to deepen our understanding of underlying molecular mechanisms, develop robust neural interfaces, and elucidate complex neural circuitry so we can better diagnose and treat conditions under our sites. As we continue to understand the human nervous system and the language it uses, we will be better able to detect early warnings of disease and to address a wide array of conditions through neural decoding and modulation. Many open questions and challenges remain in the field of bioelectronic medicine. However, recent advances have shown what is possible for patients of the future.

\section{RESTORING MOVEMENT IN A HUMAN}

Decades of research in understanding how movement information is encoded in the brain, development of chronic neural interfaces, and the development of neural decoding methods has laid the foundation for new advances and the development of bioelectronic neural bypass technology. Until 2014, restoration of cortical control of movement in a paralyzed person had not been achieved. In that year, a clinical study was launched with that goal in mind. A 24-year-old young man, who was left quadriple- gic after a diving accident, was enrolled in the study. Within the first year, he was able to regain functional use of his right hand through the use of an artificial bypass, which combined a cortical neural interface, neural decoding algorithms, and transcutaneous neuromuscular stimulation (Bouton et al. 2016). The neural implant $(4 \times 4$ $\mathrm{mm}$ across with 96 electrodes, each $1.5 \mathrm{~mm}$ long) was placed in his left primary motor cortex and was linked through a computer to a neuromuscular stimulator with $>100$ electrodes placed on his forearm (see Fig. 1). This approach allowed the study participant to regain volitional control of hand grasping and individual finger movements.

Another group of researchers showed later that a BCI, with assistance from a motorized arm, could allow volitional movement in a C4 participant (Ajiboye et al. 2017).

As shown in Figure 2, the participant was able to achieve functional movements such as picking up a cup using a cylindrical grasp and volitionally switching to a pinch grasp. This allowed him to pick up smaller objects, such as the stir stick shown in Figure 2, with finer dexterity.

\section{DECODING RHYTHMIC MOVEMENTS}

Rhythmic movements, such as scratching, brushing your teeth, or playing a musical instrument, are all activities related to a person's independence and quality of life. Neural circuits in the spinal cord and brain stem facilitate these rhythmic movements (Brown 1914; Marder and Bucher 2001). However, when they are damaged through injury or stroke, restoring volitional rhythmic movement can be particularly challenging or impossible. With a neural bypass approach, signals are rerouted around these important circuits, and, therefore, lost function must be replaced. To do this, neural circuits within the spinal cord must be emulated. These circuits are called central pattern generators (CPGs), which produce motor patterns for rhythmic movement (Marder and Bucher 2001). Recently, artificial CPGs have been created in software and linked to decoded brain activity sensed in primary motor cortex of a paralyzed human 


\section{C.E. Bouton}
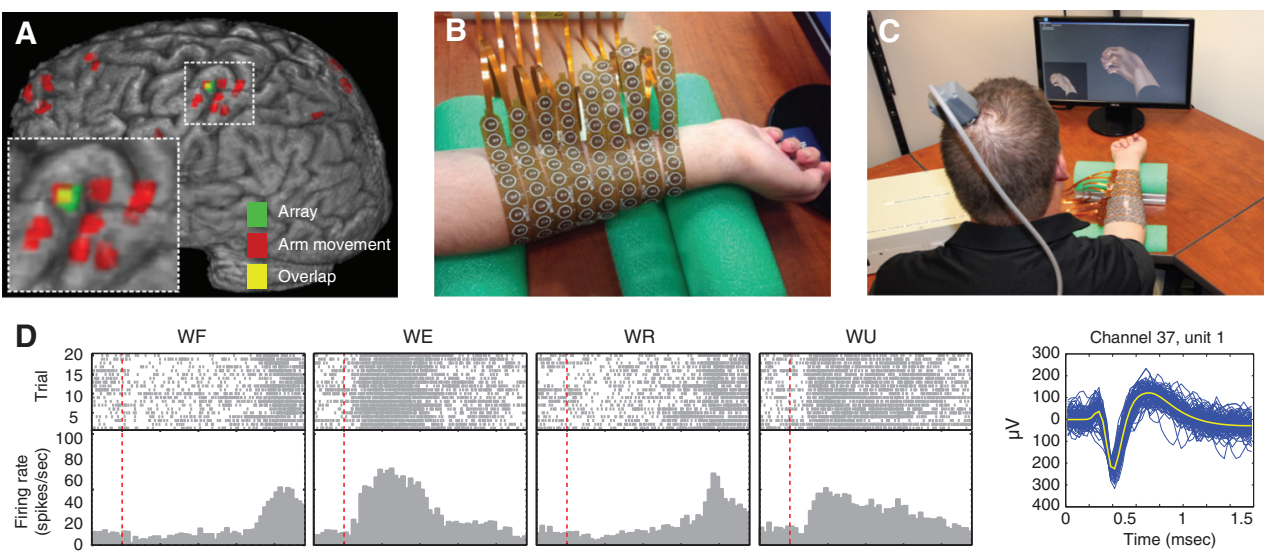

E
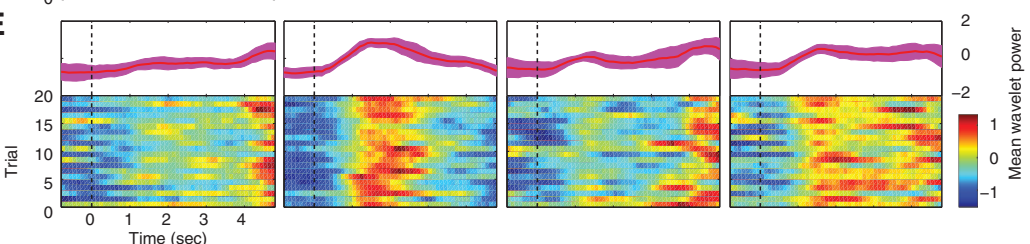

Figure 1. Brain-computer interface (BCI) system for movement restoration in a paralyzed human study participant. $(A)$ Cortical implant location, $(B)$ muscle stimulation sleeve, $(C)$ experimental setup, and $(D, E)$ neural activity for imagined/attempted wrist movements (extension, flexion, and radial/ulnar deviations). (From Bouton et al. 2016; reprinted, with permission.)

(Sharma et al. 2016). In this work, CPG behavior was emulated by using a numerical model of an oscillatory neural circuit. This circuit is shown in Figure 3.

In primates, corticomotoneurons in the primary motor cortex monosynaptically project to spinal motor neurons allowing more direct muscle control. This type of connection is readily observed in the hand and arm areas of the motor cortex, and is believed to allow highly skilled movements to be learned (Rathelot and Strick 2009). In the study involving restoration

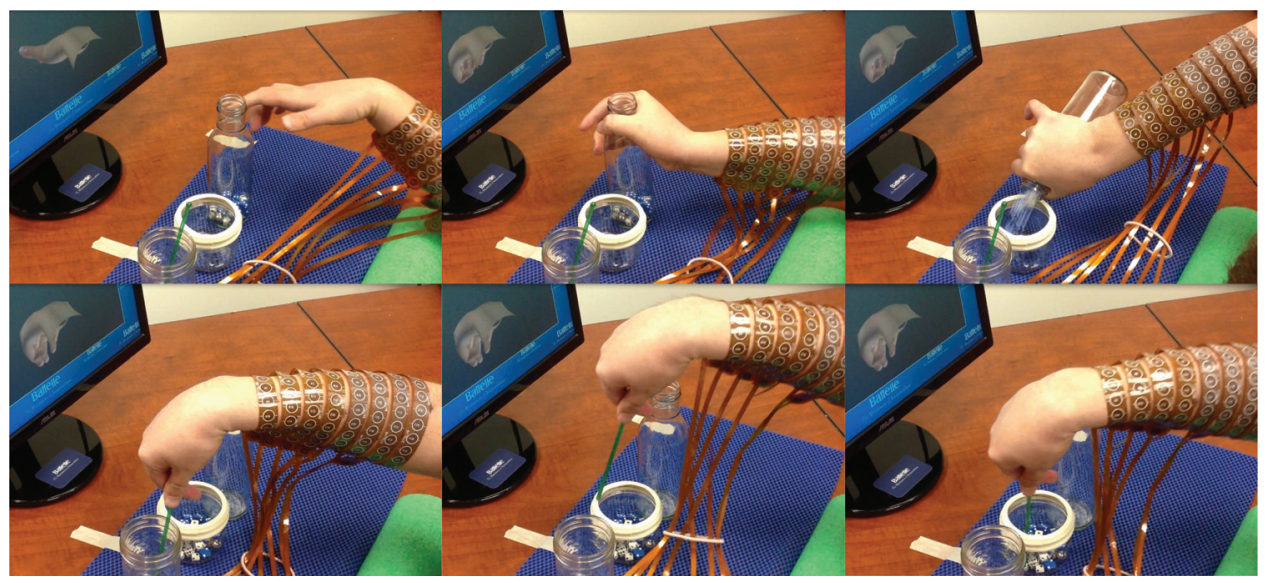

Figure 2. Functional movements achieved by a paralyzed study participant using an electronic neural bypass linking decoded brain activity to muscle activation in real time. (From Bouton et al. 2016; reprinted, with permission.) 

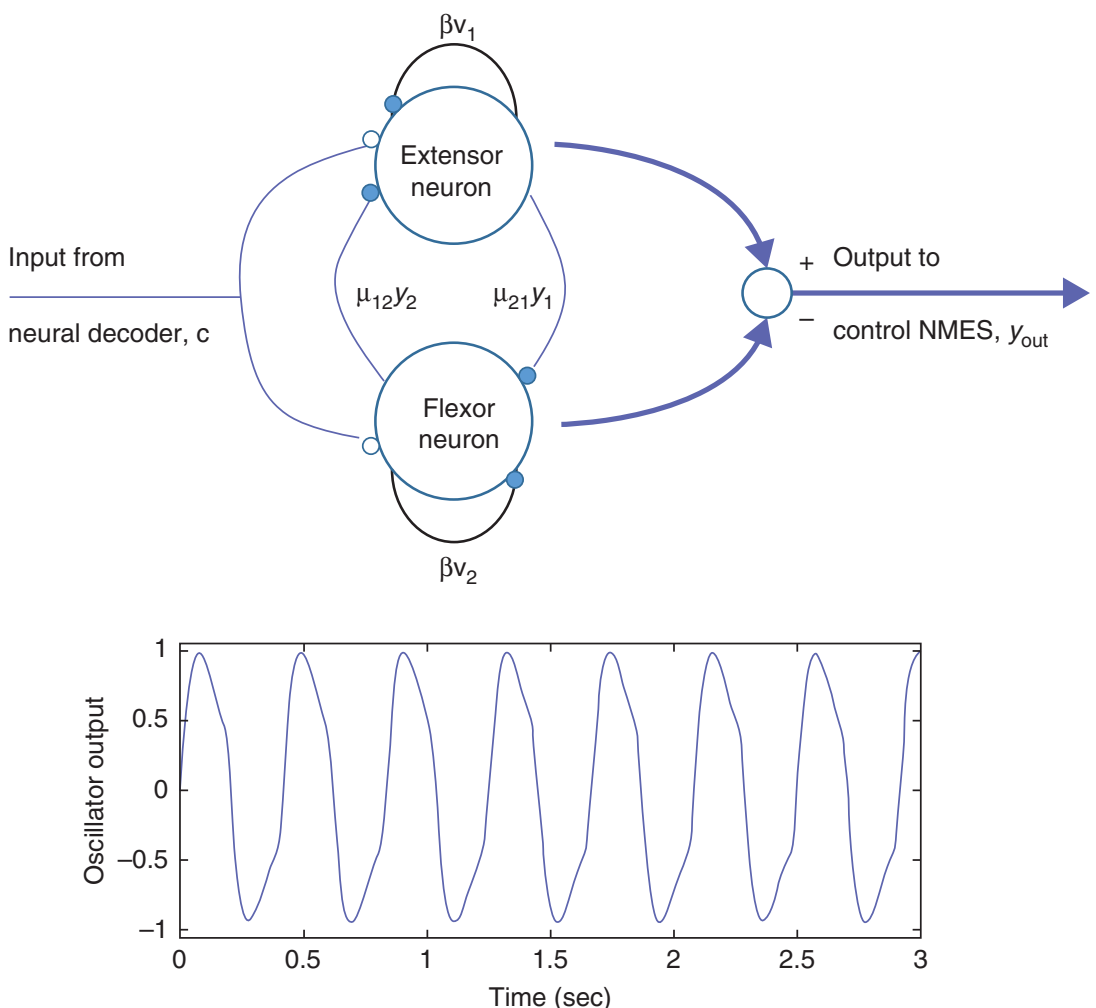

Figure 3. Two neuron oscillator models and resulting output. (From Sharma et al. 2016; reprinted, with permission, from Scientific Reports in conjunction with a Creative Commons Attribution 4.0 International License.)

of rhythmic movement in a human, it was hypothesized that two unique modulation patterns would be observed when the participant imagined a static versus rhythmic joint movement. It was found that when the participant visualized these different types of movements of the same joint, differing neuronal patterns were indeed observed. These patterns could also be accurately decoded, which allowed the participant to initiate and switch volitionally between static and rhythmic movements.

\section{CORTICAL CONTROL OF MUSCLE CONTRACTION LEVEL}

Achieving variable force for various movements is also important in our daily lives to do tasks such as holding a cup of coffee or picking up an egg. Until recently, restoring cortical control of such movements in a paralyzed person had not been achieved. In a clinical study with a quadriplegic participant previously described in Bouton et al. (2016), a new experimental paradigm was created to explore the possibility of restoring graded muscle contractions (Friedenberg et al. 2017). In this study, the participant was initially asked to imagine flexing their wrist (against a load) to different angles. The participant was not able to flex their wrist without use of the artificial neural bypass under study because of their injury. Neural activity in primary motor cortex was first recorded and decoded to allow the participant to control a virtual pointer on the computer screen. Beta regression methods were first used to decode the neural activity and identify modulation patterns of interest on specific electrode channels (1-96). As shown in Figure 4, some channels show a linear increase in activity as measured through mean wavelet power (MWP) when target angle increases. Further- 


\section{C.E. Bouton}
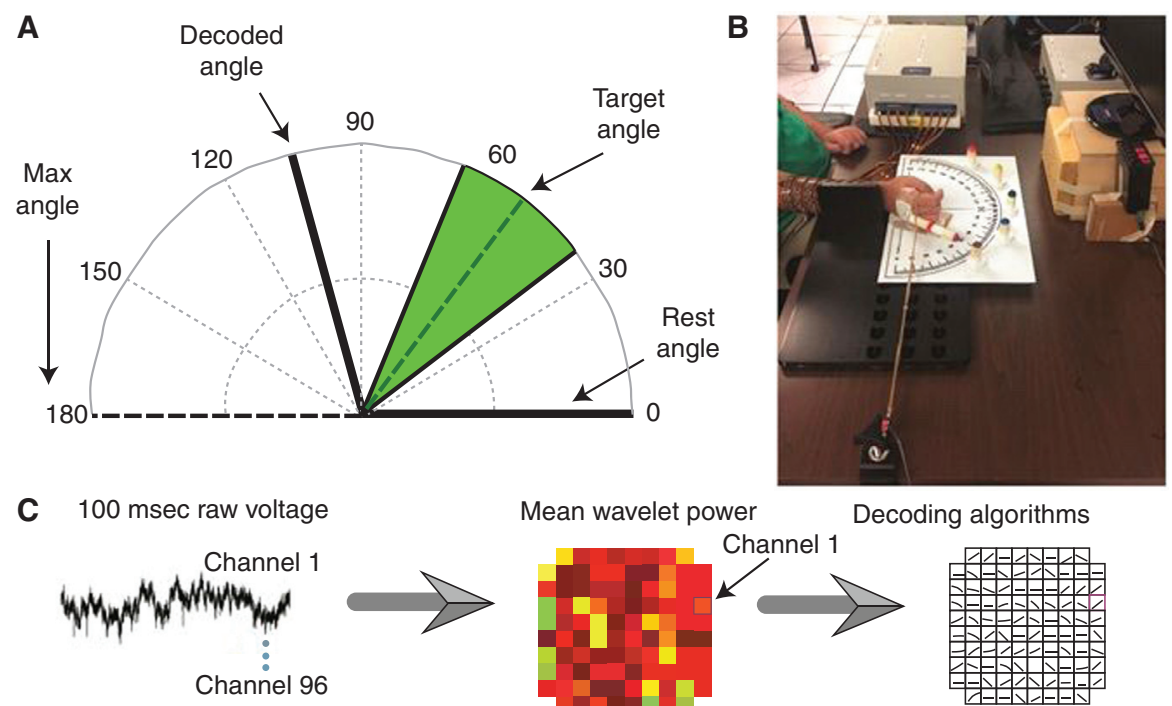

Mean wavelet power

Decoding algorithms

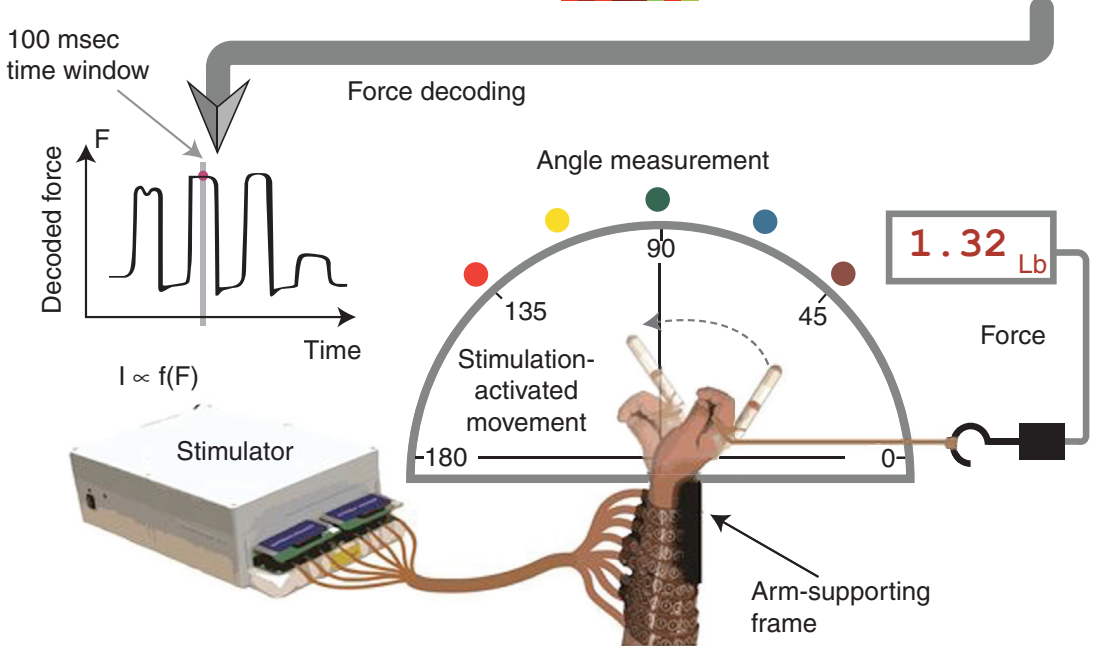

Figure 4. Decoding graded muscle contraction from intracortical activity. (A) Example screen used during experiment showing a target angle and acceptable window (green) and decoded angle (thick black line) updated in real time. $(B)$ Photograph of the setup. $(C)$ Flow chart where intracortically recorded voltage data are converted to mean wavelet power (MWP) features, then decoded to produce force, $\mathrm{F}$, and translated into a set of stimulation parameters, I, which results in wrist flexion movement recorded by the load cell and an overhead camera. (From Friedenberg et al. 2017; reprinted, with permission, from Scientific Reports in conjunction with a Creative Commons Attribution 4.0 International License.)

more, some channels show nonlinear behavior as the angle changes.

Because of the nonlinear relationship between neural activity and wrist angle, a support vector regression (SVR) method was chosen for use during reanimation of the wrist (through neuromuscular stimulation) in the physical task.
In this task, a rubber band was used to provide resistance as the participant attempted to flex his wrist to various target angles. The SVR algorithm also used a regularization parameter to "weed out" channels that were not contributing effectively to decoding. In a series of real-time tasks using the SVR algorithm, and after recal- 
ibrating the system to account for muscle fatigue, the participant was able to achieve an overall accuracy of $87.5 \% \pm 6.8 \%$.

Finally, in the study involving restoration of graded muscle contraction, it was investigated whether the participant could flex his wrist to angles that were not previously encountered during training. The rest $\left(0^{\circ}\right)$ and maximum angle were not changed, but the three target angles between were changed to new angles. The participant was successful in 14 of the 18 angle cues given and an overall accuracy of $89.6 \% \pm 4.4 \%$ was achieved.

\section{CHRONIC NEURAL RECORDINGS}

One of the outstanding challenges in the field of bioelectronic medicine is how to achieve chronic (long-term) neural recordings. Specifically, brain interfaces can be particularly challenging owing to the glial response of the brain (Williams et al. 1999). Single-unit activity (SUA) results from axonal output close to a recording electrode (Rousche and Normann 1998) and can degrade or disappear only a few months after implanting an electrode in the brain (Sharma et al. 2015) as shown in Figure 5. Multiunit activity stems from combined neuronal output from multiple neurons within several hundred microns of an electrode and has been shown to be more robust over time (Stark and Abeles 2007; Scheid et al. 2013; Sharma et al. 2015). Finally, local field potential (LFP) represents a large number of neurons firing together and typically is considered to be in the range of $<100 \mathrm{~Hz}$ and also robust over time (Scherberger et al. 2005; Sharma et al. 2015).

To combat degrading signals in chronic brain recordings, two strategies have been used. On the hardware side, thinner, more flexible electrodes made of carbon have been explored and showed a reduced inflammatory response (Guitchounts et al. 2013). A second strategy involves using signal processing methods that extract MUA features for decoding. These features have been shown to be more robust over time and still contain useful information for decoding purposes (Bouton et al. 2016). It is, however, extremely important to select the appropriate frequency range so that neighboring
A

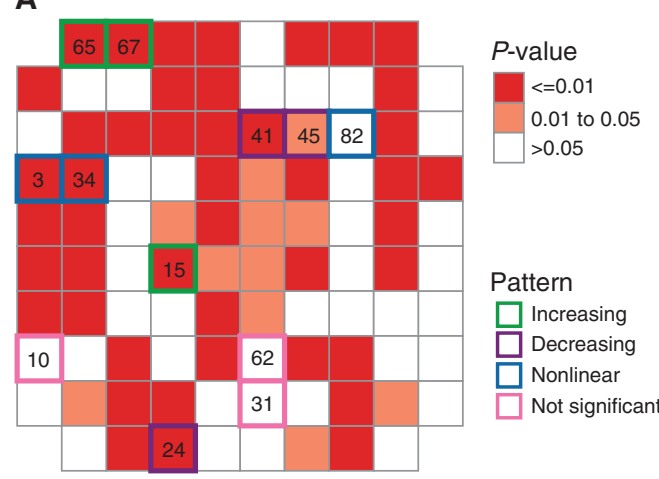

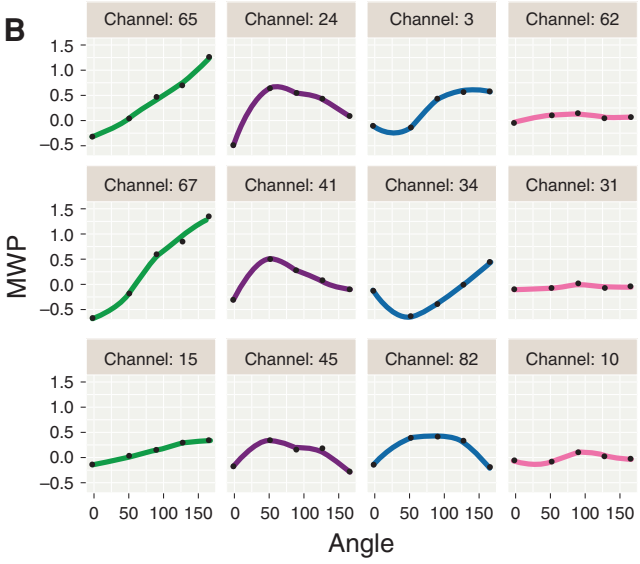

Figure 5. Neural modulation patterns with imagined graded muscle contractions. ( $A$ ) The $P$-values from the beta regression are overlaid over the physical layout of the microelectrode array (MEA). Selected individual channels (highlighted in $A$ ) shows a range of neural modulation trends in response to the cued angles. $(B)$ Each column shows selected individual channels in which the average mean wavelet power (MWP) (column 1) increased with increasing cue angles (green), (2) decreased with increasing nonzero cue angles (purple), (3) showed a nonlinear relationship with cue angle (blue), and (4) did not show significant response to the cue angles (pink). (From Friedenberg et al. 2017; reprinted, with permission, from Scientific Reports in conjunction with a Creative Commons Attribution 4.0 International License.) 


\section{C.E. Bouton}

electrodes do not contain redundant information. Because LFP, for example, results from a large number of neurons (within a larger radius around the electrode), it could be expected that the similarity of LFP signals for electrodes in close proximity to each other would be high because their large "listening radii" would overlap considerably. In fact, one might expect a general relationship (with exceptions owing to neural connections between more distant neurons) between correlation, signal frequency, and distance. This relationship has been studied (Sharma et al. 2015) through coherence analysis. It is clear that, on average, LFP signals (low frequencies) are highly correlated for neighboring electrodes, and multiunit activity (medium frequencies) and SUA (high frequencies) are much less correlated and can provide better (more independent) signals for decoding purposes for closely spaced electrodes. However, because the SUA signals have been observed to degrade over time, multiunit signals have proven to be effective and more robust for chronic decoding.

Another feature, called midfrequency MWP (mf-MWP) spanning $234 \mathrm{~Hz}$ to $3750 \mathrm{~Hz}$, has been studied in a human participant over extended time periods (Zhang et al. 2018). This feature was shown to be stable over extended periods of time and to correlate with MUA (defined as $300-6000 \mathrm{~Hz}$ in this study). Furthermore, the overall decoding accuracy for $\mathrm{mf}$ MWP features in two different tasks (involving imagined hand grasping and wrist rotation movements) was found to be $91.05 \% \pm 2.79 \%$ and $83.97 \% \pm 3.99 \%$, respectively.

\section{FEATURE SELECTION}

A critical step in the process of decoding neural signals, and in any machine learning endeavor, is feature selection and extraction. Useful features in the input data to a decoding algorithm that contain useful information must be identified. An example of a feature in neural data is firing/spiking rate (if SUA is being used). With any neural signal, the power of that signal in various frequency subbands would be another example of useful features. Various signal processing methods can be used to extract these features from data sets.

One signal processing method that can be used is bandpass filtering. This type of filter can be used to suppress unwanted components of the signal outside the frequency band of interest. After filtering is applied, a root-mean-square operation can be used to estimate the power contained in the filtered signal. Variation in this power may be useful as a feature because it may change during various decoding tasks (e.g., imagined hand movements in a motor decoding task). Furthermore, a sliding window can be used to further filter the power estimate to reduce variability. This type of filter is formed by multiplying the $N$ most recent data points in time by either a uniform set of coefficients (e.g., boxcar/moving average) or other window shapes (e.g., exponential filter).

Fast Fourier transform (FFT) filters can also be used to estimate power levels in frequency bands of interest. This approach can have a reduced temporal resolution, which may not be appropriate for transient events. In this case, wavelet techniques can be used to achieve sufficient resolution in both time and frequency domains (Graps 1995). When performing wavelet transformations, a "scale" represents a frequency range. Various scales can be used to represent SUA, MUA, and LFP activity. Wavelet coefficients can then be used as features for realtime decoding purposes (Bouton et al. 2016).

\section{NEURAL DECODING ALGORITHMS}

Once a set of features have been created, various machine learning methods can be used to construct a neural decoding algorithm. They can take on the form of a classifier that recognizes (classifies) neural data into known, discrete classes, which could be physiological states such as hypoglycemic/hyperglycemic or imagined movements someone believes about opening or closing their hand. A decoding algorithm can also perform regression in which a continuous output (prediction) is produced instead of a discrete class. 
Before a decoding algorithm can make any predictions, it must be trained. One can use supervised or unsupervised training methods. Supervised training involves the use of association to train the algorithm, the same way a person would learn a new language-we listen to the sounds for a word/phrase as someone identifies the associated object/idea. In supervised neural decoder training, neural data is collected simultaneously with label information. For example, in decoding imagined movements, the label information could be series of codes representing what the person is supposed to be imagining at each point in time. One challenge in using supervised training is that the label information/data must be synchronized with the neural data, accounting for reaction time, and it can be hard to know whether a person actually imagined what they were supposed to (they may have been distracted at various points during the training). To address this, unsupervised training can also be used (Jain et al. 1999). With this approach, algorithms automatically identify and group/cluster different neural patterns; however, each group/cluster must be identified at some point through an operator or sensor data.

Different types of machine learning methods can be used to build a classifier to regression machine. Support vector machines (SVMs) have been used for many years, and have become faster and more powerful as the computational power of devices have increased. Specific methods such as L1-SVM can be used to effectively "prune" a feature set so that only the most useful features are passed on to the decoding algorithm (Humber et al. 2010). Nonlinear/kernel methods can also be used to map neural data into a high dimensional space (Scholkopf et al. 1997). These methods can often improve decoding performance (Muller et al. 2003).

\section{REMAINING CHALLENGES AND FUTURE DIRECTIONS}

There has been tremendous progress in the field of bioelectronic medicine in recent years. However, there are critical open questions and technical challenges that must be addressed before the next generation of clinical devices can be developed. How information is encoded in the brain and the peripheral nervous system is still largely unknown. We have learned how certain types of arm and hand movements are encoded in the brain and have even learned how to decode certain movements with a respectable level of accuracy. However, there are still many questions surrounding how information is encoded and transmitted on afferent fibers in the peripheral nervous system. It has been shown that specific neural signaling occurs after exposure to interleukin (IL)-1 $\beta$ and tumor necrosis factor (TNF) (Zanos et al. 2018), but additional cytokines and resulting neural signals remain to be studied. This vast amount of information could be used for closed-loop devices, allowing automatic adjustments to stimulation and adaptation to changing conditions over time. These responsive, smart devices of the future could also lead to superior diagnostic and treatment options for patients, and, in some cases, completely replacing the need for drugs.

At the heart of a bioelectronic medicine device is the neural interface. This interface provides the critical functions of stimulating and/or recording neural signals. As previously discussed, the spatial density/spacing required for next-generation electrodes depends on the information being recorded/sensed. Currently, electrode spacing is actually quite suitable for certain decoding applications in the brain; however, the total number of electrodes and the amount of surface area being covered is lacking. For example, in motor neuroprosthetic applications for quadriplegic users, penetrating neural interfaces only cover a small portion of the hand/arm area, providing only a fraction of the information available. Increasing the total number of electrodes would allow improved decoding accuracy and/or an increased number of movements. Surface electrodes are being improved and are increasing in size; however, there is a limit to how small a single recording area can be while providing quality signals. Special coatings can combat this and further development of new materials and electrode designs will help advance efforts toward next generation neural interfaces. 


\section{C.E. Bouton}

As our knowledge in the field of bioelectronic medicine increases and new technologies are conceived and developed, a significant expansion of addressable conditions will occur. New bioelectronic devices will become more readily available and easier to apply. Noninvasive and smaller devices that are easier to implant, will also become available. These new versions will become more efficacious, adaptive, and robust over chronic period. The combination of molecular biology, neuroscience, and engineering - as a core foundation of bioelectronic medicine will continue to pave the way to a new future with many new treatment options for patients. This new approach will allow doctors to target a long list of diseases and conditions that currently have no cures, helping the millions battling paralysis, motor neuron disease, Alzheimer's, Parkinson's, traumatic brain injury, and many other debilitating conditions worldwide.

\section{REFERENCES}

Ajiboye AB, Willett FR, Young DR, Memberg WD, Murphy BA, Miller JP, Walter BL, Sweet JA, Hoyen HA, Keith MW, et al. 2017. Restoration of reaching and grasping movements through brain-controlled muscle stimulation in a person with tetraplegia: A proof-of-concept demonstration. Lancet 389: 1821-1830. doi:10.1016/S0140-6736 (17)30601-3

Bouton C. 2009. Decoding neural activity from an intracortical implant in humans with tetraplegia. In Biomedical Science \& Engineering Conference, 2009. First Annual ORNL. Oak Ridge, TN.

Bouton C. 2017. Cracking the neural code, treating paralysis and the future of bioelectronic medicine. J Int Med 282: 37-45. doi:10.1111/joim.12610

Bouton CE, Shaikhouni A, Annetta NV, Bockbrader MA, Friedenberg DA, Nielson DM, Sharma G, Sederberg PB, Glenn BC, Mysiw WJ, et al. 2016. Restoring cortical control of functional movement in a human with quadriplegia. Nature 533: 247-250. doi:10.1038/nature17435

Brown TG. 1914. On the nature of the fundamental activity of the nervous centres; Together with an analysis of the conditioning of rhythmic activity in progression, and a theory of the evolution of function in the nervous system. J Physiol 48: 18-46. doi:10.1113/jphysiol.1914.sp001646

Caravaca AS, Tsaava T, Goldman L, Silverman H, Riggott G Chavan SS, Bouton C, Tracey KJ, Desimone R, Boyden ES, et al. 2017. A novel flexible cuff-like microelectrode for dual purpose, acute and chronic electrical interfacing with the mouse cervical vagus nerve. J Neural Eng 14: 066005. doi:10.1088/1741-2552/aa7a42
Carmena JM, Lebedev MA, Crist RE, O’Doherty JE, Santucci DM, Dimitrov DF, Patil PG, Henriquez CS, Nicolelis MA. 2003. Learning to control a brain-machine interface for reaching and grasping by primates. PLoS Biol 1: e42. doi:10.1371/journal.pbio.0000042

Chapin JK, Moxon KA, Markowitz RS, Nicolelis MA. 1999. Real-time control of a robot arm using simultaneously recorded neurons in the motor cortex. Nat Neurosci 2: 664-670. doi:10.1038/10223

Cheney PD, Fetz EE. 1980. Functional classes of primate corticomotoneuronal cells and their relation to active force. J Neurophysiol 44: 773-791. doi:10.1152/jn.1980. 44.4.773

Chiluwal A, Narayan RK, Chaung W, Mehan N, Wang P, Bouton CE, Golanov EV, Li C. 2017. Neuroprotective effects of trigeminal nerve stimulation in severe traumatic brain injury. Sci Rep 7: 6792. doi:10.1038/s41598-01707219-3

Collinger JL, Wodlinger B, Downey JE, Wang W, TylerKabara EC, Weber DJ, McMorland AJ, Velliste M, Boninger ML, Schwartz AB. 2013. High-performance neuroprosthetic control by an individual with tetraplegia. Lancet 381: 557-564. doi:10.1016/S0140-6736(12)618 16-9

Donoghue JP, Sanes JN, Hatsopoulos NG, Gaál G. 1998. Neural discharge and local field potential oscillations in primate motor cortex during voluntary movements. $J$ Neurophysiol 79: 159-173. doi:10.1152/jn.1998.79.1.159

Ethier C, Oby ER, Bauman MJ, Miller LE. 2012. Restoration of grasp following paralysis through brain-controlled stimulation of muscles. Nature 485: 368-371. doi:10.1038/nature10987

Evarts EV. 1968. Relation of pyramidal tract activity to force exerted during voluntary movement. J Neurophysiol 31: 14-27. doi:10.1152/jn.1968.31.1.14

Fetz EE, Finocchio DV, Baker MA, Soso MJ. 1980. Sensory and motor responses of precentral cortex cells during comparable passive and active joint movements. J Neurophysiol 43: 1070-1089. doi:10.1152/jn.1980.43.4. 1070

Friedenberg DA, Schwemmer MA, Landgraf AJ, Annetta NV, Bockbrader MA, Bouton CE, Zhang M, Rezai AR Mysiw WJ, Bresler HS, et al. 2017. Neuroprosthetic-enabled control of graded arm muscle contraction in a paralyzed human. Sci Rep 7: 8386. doi:10.1038/s41598-01708120-9

Georgopoulos AP, Schwartz AB, Kettner RE. 1986. Neuronal population coding of movement direction. Science 233: 1416-1419. doi:10.1126/science. 3749885

Graps A. 1995. An introduction to wavelets. IEEE Comput Sci Eng 2: 50-61. doi:10.1109/99.388960

Guitchounts G, Markowitz JE, Liberti WA, Gardner TJ 2013. A carbon-fiber electrode array for long-term neural recording. J Neural Eng 10: 046016. doi:10.1088/17412560/10/4/046016

Hochberg LR, Serruya MD, Friehs GM, Mukand JA, Saleh M, Caplan AH, Branner A, Chen D, Penn RD, Donoghue JP. 2006. Neuronal ensemble control of prosthetic devices by a human with tetraplegia. Nature 442: 164-171. doi: $10.1038 /$ nature 04970

Hochberg LR, Bacher D, Jarosiewicz B, Masse NY, Simeral JD, Vogel J, Haddadin S, Liu J, Cash SS, van der Smagt P, 
et al. 2012. Reach and grasp by people with tetraplegia using a neurally controlled robotic arm. Nature 485: $372-$ 375. doi:10.1038/nature11076

Hsu JM, Rieth L, Normann RA, Tathireddy P, Solzbacher F. 2009. Encapsulation of an integrated neural interface device with Parylene C. IEEE Trans Biomed Eng 56: 23-29. doi:10.1109/TBME.2008.2002155

Humber C, Ito K, Bouton C. 2010. Nonsmooth formulation of the support vector machine for a neural decoding problem. arXiv. http://arxiv.org/abs/1012.0958v1

Jain AK, Murty N, Flynn PJ. 1999. Data clustering: A review. ACM Comput Surveys 31: 264-323. doi:10.1145/331499. 331504

Kennedy PR, Bakay RA. 1998. Restoration of neural output from a paralyzed patient by a direct brain connection. Neuroreport 9: 1707-1711. doi:10.1097/00001756-19980 6010-00007

Lebedev MA, Carmena JM, O’Doherty JE, Zacksenhouse M, Henriquez CS, Principe JC, Nicolelis MA. 2005. Cortical ensemble adaptation to represent velocity of an artificial actuator controlled by a brain-machine interface. $\mathrm{J} \mathrm{Neu-}$ rosci 25: 4681-4693. doi:10.1523/jneurosci.4088-04.2005

Lo AC, Guarino PD, Richards LG, Haselkorn JK, Wittenberg GF, Federman DG, Ringer RJ, Wagner TH, Krebs HI, Volpe BT, et al. 2010. Robot-assisted therapy for longterm upper-limb impairment after stroke. N Engl J Med 362: 1772-1783. doi:10.1056/NEJMoa0911341

Marder E, Bucher D. 2001. Central pattern generators and the control of rhythmic movements. Curr Biol 11: R986R996. doi:10.1016/S0960-9822(01)00581-4

Michie DM, Spiegelhalter DJ, Taylor CC, ed. 1994. Machine learning, neural and statistical classification. Ellis Horwood, Chichester, England.

Moritz CT, Perlmutter SI, Fetz EE. 2008. Direct control of paralysed muscles by cortical neurons. Nature 456: 639642. doi:10.1038/nature 07418

Muller KR, Anderson CW, Birch GE. 2003. Linear and nonlinear methods for brain-computer interfaces. IEEE Trans Neural Syst Rehabil Eng 11: 165-169. doi:10.1109/ TNSRE.2003.814484

Olofsson PS, Tracey KJ. 2017. Bioelectronic medicine: Technology targeting molecular mechanisms for therapy. $J$ Intern Med 282: 3-4. doi:10.1111/joim.12624

Pavlov VA, Tracey KJ. 2015. Neural circuitry and immunity. Immunol Res 63: 38-57. doi:10.1007/s12026-015-8718-1

Perkel DH, Bullock TH. 1968. Neural coding. Neurosci Res Program Bull 6: 221.

Rathelot JA, Strick PL. 2009. Subdivisions of primary motor cortex based on cortico-motoneuronal cells. Proc Natl Acad Sci 106: 918-923. doi:10.1073/pnas.0808362106

Rousche PJ, Normann RA. 1998. Chronic recording capability of the Utah Intracortical Electrode Array in cat sensory cortex. J Neurosci Methods 82: 1-15. doi:10.1016/ S0165-0270(98)00031-4

Scheid MR, Flint RD, Wright ZA, Slutzky MW. 2013. Longterm, stable behavior of local field potentials during brain machine interface use. Conf Proc IEEE Eng Med Biol Soc 2013: 307-310.

Scherberger H, Jarvis MR, Andersen RA. 2005. Cortical local field potential encodes movement intentions in the pos- terior parietal cortex. Neuron 46: 347-354. doi:10.1016/ j.neuron.2005.03.004

Scholkopf B, Sung KK, Burges CJC, Girosi F, Niyogi P, Poggio T, Vapnik V. 1997. Comparing support vector machines with Gaussian kernels to radial basis function classifiers. IEEE Trans Signal Process 45: 2758-2765. doi:10.1109/78.650102

Serruya MD, Hatsopoulos NG, Paninski L, Fellows MR, Donoghue JP. 2002. Instant neural control of a movement signal. Nature 416: 141-142. doi:10.1038/416141a

Sharma G, Annetta N, Friedenberg D, Blanco T, Vasconcelos D, Shaikhouni A, Rezai AR, Bouton C. 2015. Time stability and coherence analysis of multiunit, single-unit and local field potential neuronal signals in chronically implanted brain electrodes. Bioelectron Med 2: 63-71.

Sharma G, Friedenberg DA, Annetta N, Glenn B, Bockbrader M, Majstorovic C, Domas S, Mysiw WJ, Rezai A, Bouton C. 2016. Using an artificial neural bypass to restore cortical control of rhythmic movements in a human with quadriplegia. Sci Rep 6: 33807. doi:10.1038/ srep33807

Shavlik JW, Dietterich TG. 1990. Readings in machine learning. Morgan Kaufmann, San Mateo, CA.

Stark E, Abeles M. 2007. Predicting movement from multiunit activity. J Neurosci 27: 8387-8394. doi:10.1523/ jneurosci.1321-07.2007

Taylor DM, Tillery SI, Schwartz AB. 2002. Direct cortical control of 3D neuroprosthetic devices. Science 296: 18291832. doi:10.1126/science.1070291

Tracey KJ. 2002. The inflammatory reflex. Nature 420: 853 859. doi:10.1038/nature01321

Tracey KJ. 2015. Electronic medicine fights disease. Sci Am 312: 28-35. doi:10.1038/scientificamerican0315-28

Velliste M, Perel S, Spalding MC, Whitford AS, Schwartz AB. 2008. Cortical control of a prosthetic arm for self-feeding. Nature 453: 1098-1101. doi:10.1038/nature06996

Vetter RJ, Williams JC, Hetke JF, Nunamaker EA, Kipke DR. 2004. Chronic neural recording using silicon-substrate microelectrode arrays implanted in cerebral cortex. IEEE Trans Biomed Eng 51: 896-904. doi:10.1109/ TBME.2004.826680

Volpe BT, Lynch D, Rykman-Berland A, Ferraro M, Galgano M, Hogan N, Krebs HI. 2008. Intensive sensorimotor arm training mediated by therapist or robot improves hemiparesis in patients with chronic stroke. Neurorehabil Neural Repair 22: 305-310. doi:10.1177/ 1545968307311102

Warland DK, Reinagel P, Meister M. 1997. Decoding visual information from a population of retinal ganglion cells. $J$ Neurophysiol 78: 2336-2350. doi:10.1152/jn.1997.78.5. 2336

Wessberg J, Stambaugh CR, Kralik JD, Beck PD, Laubach M, Chapin JK, Kim J, Biggs SJ, Srinivasan MA, Nicolelis MA. 2000. Real-time prediction of hand trajectory by ensembles of cortical neurons in primates. Nature 408: 361-365. doi:10.1038/35042582

Williams JC, Rennaker RL, Kipke DR. 1999. Long-term neural recording characteristics of wire microelectrode arrays implanted in cerebral cortex. Brain Res Brain Res Protoc 4: 303-313. doi:10.1016/S1385-299X(99)00034-3 


\section{C.E. Bouton}

Zanos TP, Silverman HA, Levy T, Tsaava T, Battinelli E, Lorraine PW, Ashe JM, Chavan SS, Tracey KJ, Bouton CE. 2018. Identification of cytokine-specific sensory neural signals by decoding murine vagus nerve activity. Proc Natl Acad Sci 115: E4843-E4852. doi:10.1073/pnas.171 9083115
Zhang M, Schwemmer MA, Ting JE, Majstorovic CE, Friedenberg DA, Bockbrader MA, Mysiw WJ, Rezai AR, Annetta NV, Bouton CE, et al. 2018. Extracting wavelet based neural features from human intracortical recordings for neuroprosthetics applications. Bioelectron Med 4: 11. doi:10.1186/s42234-018-0011-x 


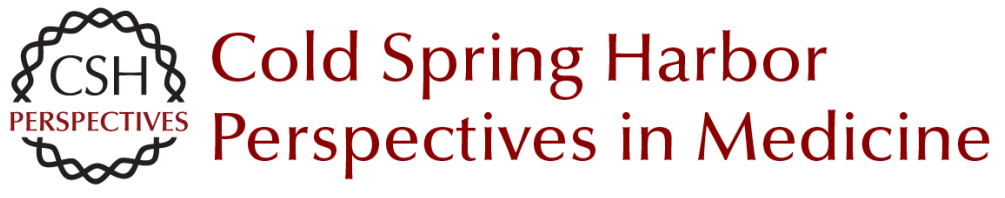

\title{
Restoring Movement in Paralysis with a Bioelectronic Neural Bypass Approach: Current State and Future Directions
}

\author{
Chad E. Bouton
}

Cold Spring Harb Perspect Med 2019; doi: 10.1101/cshperspect.a034306 originally published online February 11, 2019

Subject Collection Bioelectronic Medicine

Neural Control of Inflammation: Bioelectronic Medicine in Treatment of Chronic Inflammatory Disease

Michael Eberhardson, Laura Tarnawski, Monica Centa, et al.

Noninvasive Neuromodulation of Peripheral Nerve Pathways Using Ultrasound and Its Current

Therapeutic Implications

Christopher Puleo and Victoria Cotero

Enteric Neuromodulation for the Gut and Beyond Yogi A. Patel and Pankaj J. Pasricha

Optogenetic Control of the Peripheral Nervous System

Rui B. Chang

Closed-Loop Neuromodulation in Physiological and Translational Research

Stavros Zanos

Electrical Impedance Methods in Neuromuscular Assessment: An Overview

Seward B. Rutkove and Benjamin Sanchez

Optogenetic Medicine: Synthetic Therapeutic

Solutions Precision-Guided by Light

Haifeng Ye and Martin Fussenegger

Technobiology's Enabler: The Magnetoelectric Nanoparticle

Sakhrat Khizroev
Bioelectronic Medicine: From Preclinical Studies on the Inflammatory Reflex to New Approaches in Disease Diagnosis and Treatment

Valentin A. Pavlov, Sangeeta S. Chavan and Kevin

J. Tracey

Vagus Nerve Stimulation and the Cardiovascular System

Michael J. Capilupi, Samantha M. Kerath and

Lance B. Becker

Harnessing the Inflammatory Reflex for the

Treatment of Inflammation-Mediated Diseases

Yaakov A. Levine, Michael Faltys and David Chernoff

Recording and Decoding of Vagal Neural Signals Related to Changes in Physiological Parameters and Biomarkers of Disease Theodoros P. Zanos

Restoring Movement in Paralysis with a

Bioelectronic Neural Bypass Approach: Current

State and Future Directions Chad E. Bouton

Bioelectronic Medicine--Ethical Concerns Samuel Packer, Nicholas Mercado and Anita Haridat

Use of Bioelectronics in the Gastrointestinal Tract Larry Miller, Aydin Farajidavar and Anil Vegesna

Vagus Nerve Stimulation at the Interface of BrainGut Interactions

Bruno Bonaz, Valérie Sinniger and Sonia Pellissier

For additional articles in this collection, see http://perspectivesinmedicine.cshlp.org/cgi/collection/ 